\title{
LOCALIZATION OF EQUIVARIANT COHOMOLOGY RINGS
}

BY

J. DUFLOT

\begin{abstract}
The main result of this paper is the "calculation" of the Borel equivariant cohomology ring $H^{*}\left(E G \times{ }_{G} X, \mathbf{Z} / p \mathbf{Z}\right)$ localized at one of its minimal prime ideals. In case $X$ is a point, the work of Quillen shows that the minimal primes $\mathfrak{p}_{A}$ are parameterized by the maximal elementary abelian $p$-subgroups $A$ of $G$ and the result is
\end{abstract}

$$
H^{*}(B G, \mathbf{Z} / p \mathbf{Z})_{\mathfrak{p}_{A}} \cong H^{*}\left(B C_{G}(A), \mathbf{Z} / p \mathbf{Z}\right)_{\mathfrak{p}_{A}}^{W_{G}(A)}
$$

Here, $C_{G}(A)$ is the centralizer of $A$ in $G$, and $W_{G}(A)=N_{G}(A) / C_{G}(A)$, where $N_{G}(A)$ is the normalizer of $A$ in $G$. An example is included.

1. Introduction and preliminaries. Let $G$ be a compact Lie group and $E G \rightarrow B G$ a classifying bundle for principal $G$ bundles. When $G$ acts on a space $X$ we may form the space $X_{G}=(E G \times X) / G=E G \times{ }^{G} X$-the orbit space of $E G \times X$ under the diagonal action of $G$. Let $p$ be a fixed prime; then we may consider the equivariant cohomology ring $H_{G}^{*}(X)=H^{*}\left(X_{G}, \mathbf{Z} / p \mathbf{Z}\right)$ as a module over its commutative subring

$$
H_{G}(X)=H\left(X_{G}, \mathbf{Z} / p \mathbf{Z}\right)= \begin{cases}\bigoplus_{i \geqslant 0} H^{2 i}\left(X_{G}, \mathbf{Z} / p \mathbf{Z}\right), & p>2, \\ \bigoplus_{i \geqslant 0} H^{i}\left(X_{G}, \mathbf{Z} / p \mathbf{Z}\right), & p=2 .\end{cases}
$$

(Many of the general properties of equivariant cohomology are set forth in $[\mathbf{Q} 1, \mathbf{Q} 2, \mathbf{B}, \mathbf{B r}]$ and various other sources and we shall not list these properties here. However, in order to be consistent with the above references and to suit the purposes of this paper, some restrictions must be placed on the types of $G$-spaces that we consider: they must be Hausdorff and either compact or paracompact with finite mod- $p$ cohomological dimension (see, eg., [Q1] for a definition). In addition, we assume that every $G$-space has only a finite number of orbit types and that every orbit has a "slice" around it (see, e.g., [Br] for a discussion of these ideas).)

The main theorem of this paper (Theorem 3.2) "calculates" the localization of $H^{*}\left(X_{G}, \mathbf{Z} / p \mathbf{Z}\right)$ at every minimal prime of $H\left(X_{G}, \mathbf{Z} / p \mathbf{Z}\right)$. (These minimal primes were identified by Quillen in $[\mathbf{Q 1}, \mathbf{Q 2}]$.)

As an application of this theorem we calculate the localization of $H^{*}\left(\mathrm{GL}_{n}(\mathbf{Z} / p \mathbf{Z}), \mathbf{Z} / p \mathbf{Z}\right)$ at one of the minimal primes of $H\left(\mathrm{GL}_{n}(\mathbf{Z} / p \mathbf{Z}), \mathbf{Z} / p \mathbf{Z}\right)$.

I would like to thank Daniel Quillen for his invaluable suggestions.

Received by the editors April 5, 1983

1980 Mathematics Subject Classification. Primary 20J06, 55N25. 
2. A localization lemma. Suppose $X$ and $Y$ are two spaces on which the compact Lie group $G$ acts. When one gives $X \times Y$ the diagonal $G$-action, the projection $\pi_{X}$ : $X \times Y \rightarrow X$ is $G$-equivariant and induces $\pi_{X}^{*}: H_{G}^{*}(X) \rightarrow H_{G}^{*}(X \times Y)$. If $G_{(x, y)}$ is the isotropy group at $(x, y) \in X \times Y$, then $G_{(x, y)}$ acts on the point $x$ of $X$, inducing a map $H_{G}^{*}(X) \underset{\text { res }}{\rightarrow} H_{G_{(x, y)}}^{*}(\{x\})$. One also has a map

$$
H_{G}^{*}(X) \underset{\alpha}{\rightarrow} H_{G}^{*}(G \cdot(x, y))=H_{G}^{*}(G x \times G y)
$$

(here $G \cdot(x, y), G x, G y$, etc. denote orbits of the $G$-action) given by

$$
H_{G}^{*}(X) \stackrel{\pi_{X}^{*}}{\rightarrow} H_{G}^{*}(X \times Y) \underset{\mathrm{res}}{\rightarrow} H_{G}^{*}(G \cdot x \times G \cdot y) .
$$

Using the isomorphism $H_{G}^{*}(G \cdot(x, y)) \cong{ }_{\beta} H_{G_{(x, y)}}^{*}(\{x\})$ induced by the chain of homeomorphisms

$$
\begin{array}{cc}
E G \times G_{(x, y)} x \leftrightarrow E G / G_{(x, y)} \leftrightarrow\left(E G \times{ }^{G} G\right) / G_{(x, y)} \leftrightarrow E G \times{ }^{G}\left(G / G_{(x, y)}\right) \\
\| \\
E G_{(x, y)} \times^{G_{(x, y)}\{x\}} & E G \times{ }^{G}(G x \times G y)
\end{array}
$$

one sees immediately that there is a commutative triangle:

$$
\begin{gathered}
H_{G}^{*}(X) \stackrel{\alpha}{\rightarrow} H_{G}^{*}(G \cdot x \times G \cdot y) \\
\text { res } \searrow \\
H_{G_{(x, y)}}^{*}(\{x\})
\end{gathered}
$$

Let $S \subseteq H_{G}(X)$ be a multiplicatively closed subset. Let

$$
(X \times Y)^{S}=\left\{(x, y) \mid S \cap \operatorname{ker}\left(H_{G}^{*}(X) \stackrel{\text { res }}{\rightarrow} H_{G_{(x, y)}}^{*}(\{x\})\right)=\varnothing\right\} .
$$

Using the above triangle, we see also that

$$
(X \times Y)^{S}=\left\{(x, y) \mid S \cap \operatorname{ker}\left(H_{G}^{*}(x) \stackrel{\alpha}{\rightarrow} H_{G}^{*}(G \cdot(x, y))\right)=\varnothing\right\} .
$$

Thus $(X \times Y)^{S}$ is $G$-invariant. It is also closed; we see this as follows.

Let

$$
\begin{aligned}
\mathscr{S}(G, X) & =\left\{H \leqslant G \mid X^{H} \neq \varnothing \text { and } \exists x \in X^{H} \text { with } S^{-1} H_{H}^{*}(\{x\})=0\right\} \\
& =\left\{H \leqslant G \mid X^{H} \neq \varnothing \text { and } \forall x \in X^{H}, S^{-1} H_{H}^{*}(\{x\})=0\right\} .
\end{aligned}
$$

The set $\mathscr{S}=\mathscr{S}(G, X)$ is closed with respect to taking subconjugates; i.e., if $H \in \mathscr{S}$ and $K \leq H$, then $K \in \mathscr{S}$. For, if $K \leqslant H$, then $X^{H}$ is contained in a translate of $X^{K}$; so if $X^{H} \neq \varnothing$, then $X^{K} \neq \varnothing$. Also, the diagram

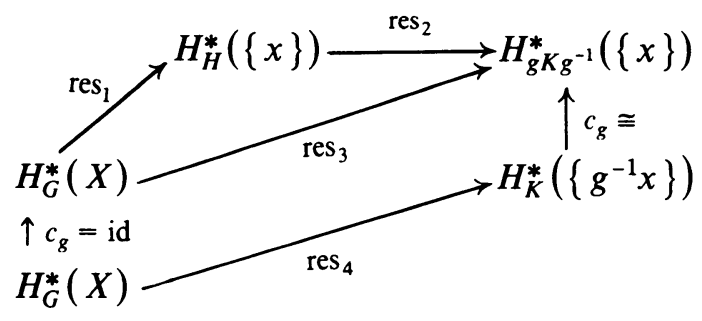


commutes, if $x \in X^{H}$ and $g \in G$; thus

$$
\begin{aligned}
S \cap \operatorname{ker}\left(\mathrm{res}_{1}\right) & \subseteq S \cap \operatorname{ker}\left(\operatorname{res}_{2} \circ \operatorname{res}_{1}\right)=S \cap \operatorname{ker}\left(\mathrm{res}_{3}\right) \\
& =S \cap \operatorname{ker}\left(c_{g}^{-1} \circ \operatorname{res}_{3}\right)=S \cap \operatorname{ker}\left(\operatorname{res}_{4}\right) .
\end{aligned}
$$

Therefore, $S^{-1}\left(H_{H}^{*}(\{x\})\right)=0$ and $S^{-1} H_{K}^{*}\left(\left\{g^{-1} x\right\}\right)=0$ if $g K g^{-1} \leqslant H$. We note that $(X \times Y)^{S}$ can be characterized as the smallest subset of $X \times Y$ with the property that every isotropy group of its complement lies in $\mathscr{S}$. Thus if $\xi \in(X \times Y)-$ $(X \times Y)^{S}$, then $G_{\xi} \in \mathscr{S}$. By the slice theorem, there is an open invariant neighborhood $U$ of $\xi$ such that $G_{u} \subseteq G_{\xi}$ for every $u \in U$; so $G_{u} \in \mathscr{S}$ for every $u \in U$. So all isotropy of $U$ is in $\mathscr{S}$; i.e. all isotropy of the complement of $(X \times Y)-U$ is in $\mathscr{S}$. Thus $(X \times Y)^{S} \subseteq(X \times Y)-U$ so $U \subseteq(X \times Y)-(X \times Y)^{S}$, proving that $(X \times Y)^{S}$ is closed.

One has the following localization result (compare with tom Dieck [T] and Hsiang [H]).

LEMMA 2.1. Suppose either:

(i) $X \times Y$ is compact and every orbit in $X$ is a G-deformation retract of one of its open neighborhoods, or

(ii) $X \times Y$ is paracompact with finite mod-p cohomology dimension.

If $Z$ is any closed invariant subset of $X \times Y$ such that all of the isotropy of $(X \times Y)-Z$ lies in $\mathscr{S}(G, X)$, then there is an isomorphism

$$
S^{-1} H_{G}^{*}(X \times Y) \stackrel{S^{-1} \text { res }}{\underset{\cong}{\rightrightarrows}} S^{-1} H_{G}^{*}(Z) .
$$

Proof. This is a straightforward modification of the standard localization theorems of, for example, tom Dieck $[\mathbf{T}]$ and Hsiang $[\mathbf{H}]$. (Note that their results are obtained by letting $Z=(X \times Y)^{S}$ and $X=\mathfrak{p t}$.)

We give the proofs of both (i) and (ii) for completeness.

(i) For every point $(x, y)$ in $X \times Y$, there is by assumption an invariant neighborhood $U$ of $G \cdot x$ and a $G$-deformation retraction $r_{1}: U \rightarrow G \cdot x$. By existence of slice, of course, there is an invariant neighborhood $V$ of $G \cdot y$ and an equivariant retraction $r_{2}: V \rightarrow G \cdot y$. Consider the following diagram (in which the diagram of solid arrows commutes):

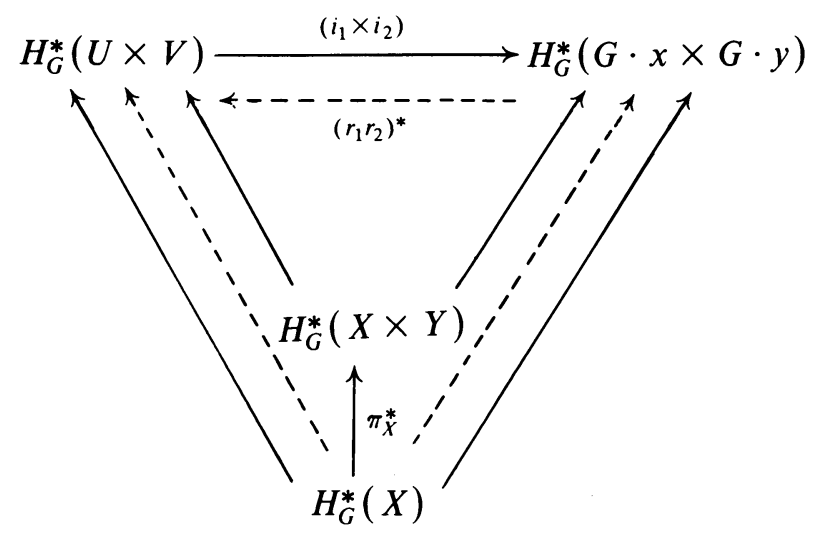


The key point in the argument is to note that the diagram of dotted arrows commutes:

$$
\begin{aligned}
\left(r_{1} \times r_{2}\right)^{*} i^{*} \pi_{X}^{*} & =\left(r_{1} \times r_{2}\right)^{*}\left(i_{2} \times i_{2}\right)^{*} j^{*} \pi_{X}^{*}=\left(i_{1} r_{1} \times i_{2} r_{2}\right)^{*} j^{*} \pi_{X}^{*} \\
& =\left(\mathrm{id} \times i_{2} r_{2}\right)^{*} j^{*} \pi_{x}^{*} \quad\left(\text { since } i_{1} r_{1} \cong{ }_{G} \mathrm{id}\right) \\
& =\left[\pi_{X} j\left(\mathrm{id} \times i_{2} r_{2}\right)\right]^{*}=\left(\pi_{X} j\right)^{*}=j^{*} \pi_{X}^{*} .
\end{aligned}
$$

Thus $\operatorname{ker} \alpha=\operatorname{ker} \alpha^{\prime}$ since $\left(r_{1} \times r_{2}\right)^{*}$ is injective. Now we use the standard arguments to prove (i).

Suppose $W$ is a closed invariant subset of $X \times Y$ with the property that all of its isotropy is in $\mathscr{S}$. For each $(x, y) \in W$, therefore, there is an $s(x, y) \in S$ such that $s(x, y) \in \operatorname{ker} \alpha$. Choosing retractive invariant neighborhoods (in $X \times Y$ ) $U_{x}, U_{y}$ (of $G \cdot x, G \cdot y$, respectively) as above we see that $S^{-1} H_{G}^{*}\left(U_{x} \times U_{y}\right)=0$ since $s(x, y) \in$ $\operatorname{ker} \alpha^{\prime}=\operatorname{ker} \alpha$. Cover $W$ by a finite number of such neighborhoods and then use a straightforward Mayer-Vietoris argument, plus exactness of localization, to find an invariant neighborhood $\mathcal{O}$ of $W$ with $S^{-1} H_{G}^{*}(\mathcal{O})=0$. Now use the continuity property (e.g. see [Q1]) and the fact that localization commutes with direct limits to show that $S^{-1} H_{G}^{*}(W)=0$.

Considering $Z$ as in the theorem, we fix a closed invariant neighborhood $V$ of $Z$ and use the above argument applied to the Mayer-Vietoris sequence for $(X \times Y)=$ $V \cup((X \times Y)-$ int $V)$ plus exactness of localization, to get

$$
S^{-1} H_{G}^{*}(V) \cong S^{-1} H_{G}^{*}(X \times Y) \text {. }
$$

Since $Z$ is closed we may let $V$ vary and again use the continuity property and the fact that localization commutes with direct limits to conclude that $S^{-1} H_{G}^{*}(X \times Y) \cong$ $S^{-1} H_{G}^{*}(Z)$.

(ii) Again, as in the proof of (i), suppose that $W$ is a closed invariant subset of $X \times Y$ with the property that all of its isotropy is in $\mathscr{S} . W$ is paracompact since it is a closed subset of $X \times Y$. Also, $W$ has finite mod- $p$ cohomological dimension. Thus the mod- $p$ cohomological dimension of $W / G$ is finite $[\mathbf{Q 1}]$ and the $E_{2}$ term of the Leray spectral sequence

$$
H^{p}\left(W / G, \mathscr{H}^{q}\right) \Rightarrow H_{G}^{p+q}(W)
$$

is bounded from the right; i.e., $E_{\mathcal{L}}^{p \cdot q}=0$ for $p>N$. Here, the stalk at $G \cdot w \in W / G$ of $\mathscr{H}^{q}$ is $H_{G s}^{q}=H_{G}^{1}(G \cdot w)$. Now, since all of the isotropy of $W$ is in $\mathscr{S}$, for each $w \in W$, one has an $s(w) \in S$ such that $s(w) \in \operatorname{ker}\left(H_{G}^{*}(X) \stackrel{\alpha}{\rightarrow} H_{G}^{*}(G w)\right)$. Since $X \times Y$ and (hence) $W$ have only a finite number of orbit types $\left\{G \cdot w_{1}, \ldots, G \cdot w_{n}\right\}$, it is not hard to see that

$$
s=s\left(w_{1}\right) \cdots s\left(w_{n}\right) \in\left[\bigcap_{n^{\prime} \in W} \operatorname{ker}\left(H_{G}^{*}(X) \rightarrow H_{G}^{*}(G \cdot w)\right)\right] \cap S .
$$

The $H_{G}^{*}(X)$-module structure on the $E_{2}$ term (given by $\pi_{X}^{*}$ and restriction on the coefficients $\mathscr{H}^{q}$ ) passes to the limit and is the same at the limit (at least up to sign) as the $H_{G}^{*}(X)$-module structure induced there by the $H_{G}^{*}(X)$-module structure $H_{G}^{*}(W)$ (again given by $\pi_{X}^{*}$ and restriction). 
Therefore $s$ maps to 0 in every stalk of the sheaf $H_{G}^{*}$ and hence maps to 0 in $H^{0}\left(W / G, \mathscr{H}^{*}\right)=E_{2}^{0, *}$ and in $E_{\infty}^{0, *}$.

Following Hsiang's argument $[\mathbf{H}]$ through, one may conclude that $s^{N+1}$ maps to zero in $H_{G}^{*}(W)$ and therefore that $S^{-1} H_{G}^{*}(W)=0$. We now finish the argument exactly as in case (i). Q.E.D.

From now on, we assume that $X$ is either compact and every orbit of $X$ is a $G$-deformation retract of one of its neighborhoods, or that $X$ is paracompact with finite mod- $p$ cohomological dimension.

3. The localization of $H_{G}^{*}(X, \mathbf{Z} / p \mathbf{Z})$ at the minimal primes of $H_{G}(X, \mathbf{Z} / p \mathbf{Z})$. The minimal primes of $H_{G}(X, \mathbf{Z} / p \mathbf{Z})$ were described by Quillen $[\mathbf{Q 1}, \mathbf{Q 2}]$ as follows. An elementary abelian p-subgroup or p-torus in $G$ is a subgroup $A$ of $G$ which is a direct product of cyclic groups of order $p$. The number of cyclic factors is the rank of $A$.

Let $\mathscr{A}(G, X)$ denote Quillen's category of pairs [Q2] $(A ; c)$ where $A$ is a $p$-torus in $G$ and $C$ is a (nonempty) connected component of $X^{A}$ (the fixpoint set of the $A$-action on $X)$; i.e., the objects of $\mathscr{A}(G, X)$ are the pairs $(A, c)$ as above, and a morphism $\theta:(A, c) \rightarrow\left(A^{\prime}, c^{\prime}\right)$ is a conjugation of $A$ into (a subgroup of) $A^{\prime}$ by an element $g$ of $G$ such that $c^{\prime} \subseteq g c$ as well. The objects of $\mathscr{A}(G, X)$ are partially ordered; i.e., $(A, c) \leqslant\left(A^{\prime}, c^{\prime}\right)$ if and only $A \leqslant A^{\prime}$ and $c$ is the unique component of $X^{A}$ containing $C^{\prime}$. We say that $(A, c)$ is subconjugate to $\left(A^{\prime}, c^{\prime}\right)$ (written $\left.(A, c) \lesssim\left(A^{\prime}, c^{\prime}\right)\right)$ if $\operatorname{Hom}_{\mathscr{A}(G, X)}\left((A, c),\left(A^{\prime}, c^{\prime}\right)\right) \neq \varnothing$; and $(A, c)$ is conjugate to $\left(A^{\prime}, c^{\prime}\right)\left((A, c) \cong\left(A^{\prime}, c^{\prime}\right)\right)$ if $(A, c)$ and $\left(A^{\prime}, c^{\prime}\right)$ are isomorphic objects.

For every pair $(A, c)$ in $\mathscr{A}(G, X)$, there is a prime ideal $\mathfrak{p}_{(A, c)} \subseteq H\left(X_{G}, \mathbf{Z} / p \mathbf{Z}\right)$; namely

$$
\mathfrak{p}_{(A, c)}=\operatorname{ker}\left(H_{G}(X) \stackrel{\text { res }}{\rightarrow} H_{A}(\mathfrak{p t}) / \sqrt{0}\right) ;
$$

where $p t \in X^{A}$ is any point in $X^{A}$. One has

THEOREM 3.1 (Proposition 11.2 OF [Q2]). (i) $\mathfrak{p}_{(A, c)} \supseteq \mathfrak{p}_{\left(A^{\prime}, c^{\prime}\right)}$ if and only if $(A, c) \leqq\left(A^{\prime}, c^{\prime}\right)$; in particular, $\mathfrak{p}_{(A, c)}=\mathfrak{p}_{\left(A^{\prime}, c^{\prime}\right)}$ if and only if $(A, c) \cong\left(A^{\prime}, c^{\prime}\right)$.

(ii) There is a one-to-one correspondence between conjugacy classes of maximal pairs $(A, c)$ and minimal prime ideals of $H_{G}(X)$ :

$$
[(A, c)] \rightsquigarrow \mathfrak{p}_{(A, c)} \text {. }
$$

One may define (as in [Q2]) $C_{G}(A, c)=\{g \in G \mid g a=a g \forall a \in A$ and $c=c\}$; $N_{G}(A, c)=\left\{g \in G \mid g A g^{-1}=A\right.$ and $\left.g c=c\right\}$, and $W_{G}(A, c)=W_{G}(A, c)=$ $N_{G}(A, c) / C_{G}(A, c)$. There is a natural action of $W_{G}(A, c)$ on $H_{C_{G}(A, c)}^{*}(c)$; we may consider both the invariant subring $H_{C_{G}(A, c)}^{*}(c)^{W_{G i}(A, C)}$, of this action, and the ring $H_{N_{G}(A, c)}^{*}(c)$ as $H_{G}^{*}(X)$-modules via restriction. Hence, we may localize all of these modules with respect to the prime $\mathfrak{p}_{(A, c)}$ of $H_{G}(X)$. The main theorem of this paper is

THEOREM 3.2. Suppose that $(A, c)$ is a maximal pair of $\mathscr{A}(G, X)$. Then there is an isomorphism

$$
H_{G}^{*}(X)_{b_{(A, C)}} \stackrel{(\mathrm{res})_{v_{(A, C)}}}{\longrightarrow} H_{C_{(i,}(A, c)}^{*}(c)_{H_{(A, C)}}^{W_{(i,}(A, c)}
$$


We note that $\operatorname{res}\left(H_{G}^{*}(X)\right) \subseteq H_{C_{G}(A, c)}^{*}(c)^{W_{G}(A, c)}$ since inner automorphisms act trivially on equivariant cohomology (e.g., see $[\mathbf{Q} 1])$, so $C_{G}(A, c)$ acts trivially on $H_{G}^{*}(X)$.

We must first prove some preliminary lemmas. Fix an embedding of $G$ in a unitary group $U$; let $S$ be the "diagonal" $p$-torus of $U$ and let $F=U / S$ be the compact smooth $G$-manifold of right cosets of $S$ in $U$.

Lemma 3.3. Suppose that $(A, c)$ is a maximal pair in $\mathscr{A}(G, X)$. Let $K$ be a closed subgroup of $G$, and let $W$ be a closed $K$-invariant subset of $X$ such that $(A, c)$ is also an object in the category $\mathscr{A}(K, W)$. Then there are localization isomorphisms for every $i \geqslant 1$,

$$
S^{-1} H_{K}^{*}\left(W \times F^{i}\right) \underset{\cong}{\rightarrow} S^{-1} H_{K}^{*}\left(G \cdot\left((c \cap w) \times\left(F^{A}\right)^{i}\right)\right),
$$

where $S=\theta\left(H_{G}(X)-\mathfrak{p}_{(A, c)}\right)$ and $\theta$ is the restriction $H_{G}(X) \rightarrow H_{K}(W)$.

Moreover, if one also knows that any pair in $\mathscr{A}(K, W)$ equivalent to $(A, c)$ in $\mathscr{A}(G, X)$ is also equivalent to $(A, c)$ in $\mathscr{A}(K, W)$ (we then say $(A, c)$ is weakly closed in $\mathscr{A}(K, W)$ with respect to $\mathscr{A}(G, X))$, then there are isomorphisms for every $i \geqslant 1$,

$$
S^{-1} H_{K}^{*}\left(W \times F^{i}\right) \stackrel{\cong}{\rightarrow} S^{-1} H_{K}^{*}\left(K \cdot\left(c \times\left(F^{A}\right)^{i}\right)\right) .
$$

Proof. We apply the localization theorem to $W$ (not $X$ ) with $Y=F^{i}$. ( $W$ inherits the necessary properties of $X$ since it is closed. $W \times F^{i}$ then has the necessary properties since $F^{i}$ is a compact manifold.)

We need to verify

(i) that $G \cdot\left(c \cap W \times\left(F^{A}\right)^{i}\right)$ and $K \cdot\left(c \times\left(F^{A}\right)^{i}\right)$ are closed $K$-invariant subsets of $W \times F^{i}$ and

(ii) that 11 of the isotropy of the complement of $G \cdot\left(c \cap W \times\left(F^{A}\right)^{i}\right)$ (or of $K \cdot\left(c \times\left(F^{A}\right)^{i}\right)$ in case $(A, c)$ is weakly closed $)$ is in

$$
\mathscr{S}(K, W)=\left\{K^{\prime} \leqslant K \mid W^{K^{\prime}} \neq \varnothing \text { and } S^{-1} H_{K^{\prime}}^{*}(\{w\})=0 \forall w \in W^{K^{\prime}}\right\} .
$$

(i) is straightforward.

To show (ii), suppose that $\xi \in W \times F^{i}$ and $K_{\xi} \notin \mathscr{S}(K, W)$. Let $\xi=\left(x, f_{1}, \ldots, f_{i}\right)$. Then $K_{\xi}=K_{x} \cap K_{f_{1}} \cap \cdots \cap K_{f_{i}}=B$ is a $p$-torus in $K$ since all isotropy groups of $F$ are $p$-tori [Q1]. Also, $x \in W^{B}$ since $K_{x} \geqslant K_{\xi}=B$. Let us suppose that $x$ lies in the component $d$ of $W^{B}$, which in turn lies in the component $c^{\prime}$ of $X^{B}$.

Since $K_{\xi} \notin \mathscr{S}(K, W), S^{-1} H_{B}^{*}(\{x\}) \neq 0$; i.e. $S \cap \operatorname{ker}\left(H_{k}^{*}(W) \rightarrow H_{B}^{*}(\{x\})\right)=\varnothing$.

But this means that

$$
S \cap \operatorname{ker}\left(H_{K}(W) \rightarrow H_{B}(x \in d) / \sqrt{0}=H_{B}\left(x \in c^{\prime}\right) / \sqrt{0}\right)=\varnothing .
$$

Consider the following commutative diagram of restrictions:

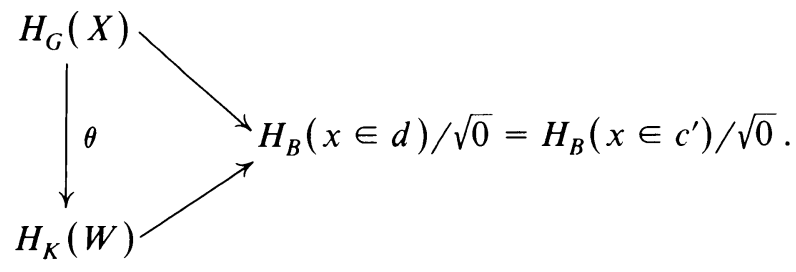


Since $\theta\left(H_{G}(X)-\mathfrak{p}_{(A, c)}\right) \cap \operatorname{ker}\left[H_{K}(W) \rightarrow H_{B}(x \in d) / \sqrt{0}\right]=\varnothing$ the above diagram shows that $\left[H_{G}(X)-\mathfrak{p}_{(A, c)}\right] \cap \operatorname{ker}\left(H_{G}(X) \rightarrow H_{B}(x \in x) / \sqrt{0}\right)=\varnothing$. Thus $\mathfrak{p}_{\left(B, c^{\prime}\right)} \subseteq \mathfrak{p}_{(A, c)}$ in $H_{G}(X)$. Therefore, since $\mathfrak{p}_{(A, c)}$ is minimal [Q2], $\mathfrak{p}_{\left(B, c^{\prime}\right)}=\mathfrak{p}_{(A, c)}$. But then $\left(B, c^{\prime}\right) \cong(A, c)$ in $\mathscr{A}(G, X)[\mathbf{Q} 2]$. By definition, this means that there is some $g \in G$ such that $g B g^{-1}=A$ and $g c^{\prime}=c$. Since $x \in c^{\prime}, x \in g c^{\prime}=c$; and since $f_{j} \in F^{B}$ for each $j$ (since $B \subseteq K_{f_{j}}$ for every $j$ ), we see that $f g_{j} \in g F^{B}=F^{g B g^{-1}}=F^{A}$ so that $g \xi \in c \times\left(F^{A}\right)^{i}$ and thus that $\xi \in(G c \cap W) \times G \cdot\left(F^{A}\right)^{i}$.

For $(* *)$, we simply note that if the $g$ in the paragraph above may always be chosen from $K$, then $K_{\xi} \notin \mathscr{S}(K, W)$ implies that $\xi \in K \cdot\left(c \times\left(F^{A}\right)^{i}\right)$. Q.E.D.

LEMMA 3.4. If $(A, c)$ is a maximal pair in $(G, X)$, then there are isomorphisms for $i \geqslant 1$,

$$
H_{G}^{*}\left(G \cdot\left(c \times\left(F^{A}\right)^{i}\right)\right) \stackrel{\cong}{\rightarrow} H_{N_{G}(A, c)}^{*}\left(c \times\left(F^{A}\right)^{i}\right) .
$$

Proof. This follows from the fact there is a $G$-equivariant homeomorphism

$$
G \times{ }^{N_{G}(A, c)}\left[c \times\left(F^{A}\right)^{i}\right] \stackrel{\theta}{\rightarrow} G \cdot\left(c \times\left(F^{A}\right)^{i}\right),
$$

where $\theta\left(\left[g,\left(x, f_{1}, \ldots, f_{i}\right)\right]\right)=\left(g x, g f_{1}, \ldots, g f_{i}\right)$. Here, $N_{G}(A, c)$ acts on $G$ by $g \stackrel{n}{\mapsto} g n^{-1}$; and on $c \times\left(F^{A}\right)^{i}$ diagonally on the left: $\left(x, f_{1}, \ldots, f_{i}\right) \stackrel{n}{\mapsto}\left(n x, n f_{1}, \ldots, n f_{i}\right)$. The map $\theta$ is clearly surjective, continuous and open. If $\left(g x, g f_{1}, \ldots\right)=\left(g^{\prime} x^{\prime}, g^{\prime} f_{1}^{\prime}, \ldots\right)$ where $x$ and $x^{\prime}$ are in $c$, and $f_{1}, \ldots ; f_{1}^{\prime}, \ldots$; are in $F^{A}$, then $g x=g^{\prime} x^{\prime}$ and $g f_{j}=g^{\prime} f_{j}^{\prime}$. Thus if $h=g^{-1} g^{\prime}, h x^{\prime}=x$ and $h f_{1}^{\prime}=f_{1}, \ldots$, etc., so that $G_{x}=h G_{x^{\prime}} h^{-1}$ and $G_{f_{1}}=h G_{f_{1}^{\prime}} h^{-1}$. Now, since $x \in c \subseteq X^{A}$ and $f_{1} \in F^{A}, G_{x} \supseteq A$ and $G_{f_{1}} \supseteq A$. So $A \subseteq G_{x} \cap G_{f_{1}}$. Since all isotropy of $F$ is $p$-toral, $G_{f_{1}}$ is a $p$-torus of $G$; thus $B=G_{x} \cap G_{f_{1}}$ is a $p$-torus in $G$. Since $B$ fixes $x$ and $A \subseteq B, X^{B} \subseteq X^{A}$ and thus there is a component $d$ of $X^{B}$ containing $x$ and contained in $c$. Thus $(A, c) \subseteq(B, d)$ in $\mathscr{A}(G, X)$, so by maximality, $(A, c)=(B, d)$.

Similarly, if $B^{\prime}=G_{X^{\prime}} \cap G_{f_{1}^{\prime}}$ we see that $(A, c)=\left(B^{\prime}, d^{\prime}\right)$ where $d^{\prime}$ is a component of $X^{B^{\prime}}$ containing $x$ and contained in $c$. Thus $B=B^{\prime}=A$ and $d=d^{\prime}=c$. But $h B^{\prime} h^{-1} B$ and $h d^{\prime}=d$, so $h A h^{-1}=A$ and $h c=c$. Therefore, $h \in N_{G}(A, c)$ and clearly

$$
\left[\left(g h, h^{-1} x, \ldots, h^{-1} f_{i}\right)\right]=\left[\left(g^{\prime}, x^{\prime}, f_{1}^{\prime}, \ldots, f_{i}^{\prime}\right)\right]
$$

in $G \times{ }^{N_{G}(A, c)}\left(c \times\left(F^{A}\right)^{i}\right)$. So $\theta$ is a homeomorphism yielding a homeomorphism:

$$
\begin{aligned}
& E G \times{ }^{G}\left(G \times N_{G}(A, c)\left[c \times\left(F^{A}\right)^{i}\right]\right) \stackrel{\theta_{G}}{\rightarrow} E G \times{ }^{G}\left(G \cdot\left(c \times\left(F^{A}\right)^{i}\right)\right) \\
& \| \\
& {\left[E G \times{ }^{G} G\right] \times{ }^{N_{G}(A, c)}\left[c \times\left(F^{A}\right)^{i}\right]} \\
& \| \\
& E G \times{ }^{N_{G}(A, c)}\left[c \times\left(F^{A}\right)^{i}\right]=E\left(N_{G}(A, c)\right) \times \times^{N_{G}(A, c)}\left[c \times\left(F^{A}\right)^{i}\right] . \quad \text { Q.E.D. }
\end{aligned}
$$


Before we proceed we must look more closely at the manifold $F$ and its submanifolds $F^{B}$ where $B$ is a $p$-torus of $G$. Recall that $F=U(n) / S(n)$ where $U(n)$ is a unitary group (for an $n$-dimensional complex vector space) in which $G$ is embedded. The diagonal $p$-torus of $U(n)$ is $S(n)$. Thus a point in $F=U / S$ may be described as a pair $\left\{\left(l_{1}, \ldots, l_{n}\right) ;\left(v_{1}^{*}, \ldots, v_{n}^{*}\right)\right\}$ where $\left(l_{i}\right)$ is an ordered $n$-tuple of mutually orthogonal lines and $v_{i}^{*}$ is the orbit of a vector $v_{i}$ in the unit sphere $S^{1}\left(l_{i}\right)$ in the line $l_{i}$ under the action of the group of $p$ th roots of unity in this sphere. The action of $G$ on $F$ is via the embedding in $U$; i.e., one thinks of $g \in G$ as a unitary transformation of $C^{n}$, thus

$$
g \cdot\left\{\left(l_{n}, \ldots, l_{n}\right) ;\left(v_{1}^{*}, \ldots, v_{n}^{*}\right)\right\}=\left\{\left(g l_{1}, \ldots, g l_{n}\right) ;\left(\left(g v_{1}\right)^{*}, \ldots,\left(g v_{n}\right)^{*}\right)\right\} .
$$

(This makes sense, since $g$ preserves lengths and angles - the action of the group of $p$ th roots of unity is given by rotation through a fixed angle.)

Now, suppose that $A$ is a $p$-torus in $G$. The embedding $A \hookrightarrow G \hookrightarrow U$ gives us a unitary representation of $A$ on $\mathbf{C}^{n}$ (the restriction of the representation $G \hookrightarrow U$ of $G)$. This representation may be decomposed according to a set of distinct $n$-dimensional complex irreducible characters $\left\{\chi_{1}, \ldots, \chi_{k}\right\}$ of $A$. If we let $V_{j}$ denote the eigenspace of the character $\chi_{j}$ and $n_{j}=\operatorname{dim} V_{j}$, then we have an orthogonal decomposition of $\mathbf{C}^{n}$,

$$
\mathbf{C}^{n}=V_{1} \perp \cdots \perp V_{k} \text { and } n=n_{1}+\cdots+n_{k} .
$$

(We assume that $n_{j}>0$ for every $j$.)

Let $\Sigma\left(n_{1}, \ldots, n_{k}\right)$ be the set of $\left(n_{1}, \ldots, n_{k}\right)$-shuffles of $\{1,2, \ldots, n\}$;

$$
\begin{array}{r}
\Sigma\left(n_{1}, \ldots, n_{k}\right)=\left\{\sigma \in \Sigma(n) \mid \sigma\left(i_{11}, i_{12}, \ldots, i_{1 n_{1}}, i_{21}, \ldots, i_{2 n_{2}}, \ldots, i_{k 1}, \ldots, i_{k n_{k}}\right)\right. \\
\text { and } \left.i_{j 1}<i_{j 2}<\cdots<i_{j n_{j}}, \forall j \in\{1,2, \ldots, k\}\right\} .
\end{array}
$$

We claim that

$$
F^{A}=\underset{\sigma \in \Sigma\left(n_{1} \ldots, n_{k}\right)}{\amalg} \sigma \cdot\left(F\left(V_{1}\right) \times \cdots \times F\left(V_{k}\right)\right) .
$$

Here, $F\left(V_{i}\right)$ is the flag space for $V_{i}$, i.e., $F\left(V_{i}\right)$ consists of the pairs

$$
\left\{\left(l_{n_{1}+\cdots+n_{t-1}+1}, \ldots, l_{n_{1}+\cdots+n_{l}}\right) ;\left(v_{n_{1}+\cdots+n_{1}+1}^{*}, v_{n_{1}+\cdots+n_{t}}^{*}\right)\right\}
$$

where the $l_{j}$ 's are an ordered set of $n_{i}$ orthogonal lines in $V_{i}$, and $V_{j}^{*}$ 's are orbits of vectors $V_{j}$ in the unit spheres of the $l_{j}$ 's under the group(s) of $p$ th roots of unity in these spheres.

It should be clear what subset of $F$ that $\sigma \cdot\left(F\left(V_{1}\right) \times \cdots \times F\left(V_{k}\right)\right)$ denotes: if $\sigma=\left(i_{11}, \ldots, i_{1 n_{1}} ; i_{12}, \ldots, i_{2 n_{2}} ; \ldots ; i_{k 1}, \ldots, i_{k n_{k}}\right)$, then

$$
\begin{aligned}
\boldsymbol{\sigma} \cdot\left\{(\underbrace{l_{1}, \ldots, l_{n_{1}}}_{\text {in } V_{1}} ; \underbrace{\left.l_{n_{1}+1}, \ldots, l_{n_{1}+n_{2}} ; \ldots\right)}_{\text {in } V_{2}} ;\left(v_{1}^{*}, \ldots, v_{n_{1}}^{*} ; \ldots\right)\}\right. \\
=\left\{\left(l_{i_{11}}, l_{i_{12}}, \ldots, l_{i_{1}} ; l_{i_{21}}, \ldots, l_{i_{2 n}} ; \ldots\right) ;\left(v_{i_{11}}^{*}, \ldots, v_{i_{1_{1}}}^{*} ; \ldots\right)\right\} .
\end{aligned}
$$

Note that if $\sigma \neq \tau$ then $\sigma \cdot\left(F\left(V_{1}\right) \times \cdots \times F\left(V_{k}\right)\right) \cap \tau \cdot\left(F\left(V_{1}\right) \times \cdots \times F\left(V_{k}\right)\right)$ is indeed empty. 
Since every line represented in the direct sum on the right in equation $(*)$ is an eigenspace for some character $\chi_{\alpha^{\prime}}$, this line (and every orbit in its unit sphere) is fixed by everything in $A$. On the other hand, if an ordered collection of lines (and orbits) in $F$ are fixed by everything in $A$, then each line must be an eigenspace for the $A$-action, thus contained in some $V_{i}$. So equality (*) holds.

Since $F\left(V_{1}\right) \times \cdots \times F\left(V_{k}\right)$ is clearly connected and closed and each $\sigma \cdot\left(F\left(V_{1}\right)\right.$ $\left.\times \cdots \times F\left(V_{k}\right)\right)$ is homeomorphic to $F\left(V_{1}\right) \times \cdots \times F\left(V_{k}\right)$, the connected components of $F^{A}$ are the $\sigma \cdot\left(F\left(V_{1}\right) \times \cdots \times F\left(V_{k}\right)\right)$.

Now, $C_{G}(A)$ fixes each component of $F^{A}$. It is enough to show that $C_{G}(A)$ fixes $F\left(V_{1}\right) \times \cdots \times F\left(V_{k}\right)$. For this, we need only show that if $l$ is a line in $V_{j}$ then $g l$ is also a line in $V_{j}$ if $g \in C_{G}(A)$. (The orbits are carried along without difficulty.) Let $l=\mathbf{C} \cdot \vec{x}$ where $\vec{x}$ is a vector in $V_{j}$. Then $g \vec{x}$ is an eigenvector for $\chi_{j}: b \cdot g \vec{x}=g b \vec{x}=$ $g \cdot \chi_{j}(b) \cdot \vec{x}=\chi_{j}(b) \cdot g \vec{x}$, if $b \in A$.

Conversely, we want to show that if $g \in N_{G}(A)$, and $g$ fixes a component of $F^{A}$, then $g \in C_{G}(A)$. Again, it is enough to show this for the component $F\left(V_{1}\right) \times \cdots \times$ $F\left(V_{k}\right)$, so let $g \in N_{G}(A)$ be such that

$$
g \cdot\left(F\left(V_{1}\right) \times \cdots \times F\left(V_{k}\right)\right)=F\left(V_{1}\right) \times \cdots \times F\left(V_{k}\right) .
$$

Then $g$ carries lines in $V_{j}$ to lines in $V_{j} \forall j$, so if $\vec{x} \in V_{j}, g \vec{x} \in V_{j}$ and then $b \cdot g \vec{x}=\chi_{j}(b) g \vec{x}=g \cdot \chi_{j}(b) \vec{x}=g \cdot b \vec{x}$ for every $b \in A$, so $b^{-1} g^{-1} b g$ fixes every vector in every $V_{j}$. But $G \hookrightarrow U$ so that $b^{-1} g^{-1} b g=\mathrm{id}=\Rightarrow b g=g b$ and $g \in C_{G}(A)$. (One needs $g \in N_{G}(A)$ to ensure that $g \cdot F^{A} \subseteq F^{A}$.) Thus one sees from the above picture of $F^{A}$ that $W_{G}(A)=N_{G}(A) / C_{G}(A)$ acts freely on $\pi_{0}\left(F^{A}\right)$. Using this picture of $F^{A}$ (pointed out to me by Quillen, who also pointed out the following lemma) one may conclude

LEMMA 3.5. If $(A, c)$ is a pair in $\mathscr{A}(G, X)$ then $H_{C_{G}(A, c)}^{q}\left(c \times\left(F^{A}\right)^{i}\right)$ is a free $Z / p Z\left[W_{G}(A, c)\right]$-module for every $q \geqslant 0$ and $i \geqslant 1$.

Proof. $W_{G}(A, c)$ acts freely on $\pi_{0}\left(c \times\left(F^{A}\right)^{i}\right)=\{c\} \times \pi_{0}\left(F^{A}\right)^{i}$, so it acts freely on

$$
H_{C_{G}(A, c)}^{q}\left(c \times\left(F^{A}\right)^{i}\right)=\underset{\xi \in \pi_{0}\left(c \times\left(F^{A}\right)^{i}\right)}{\bigoplus} H_{C_{G}(A, c)}^{q}(\xi) \text {. Q.E.D. }
$$

LEMMA 3.6. If $(A, c)$ is a pair in $\mathscr{A}(G, X)$ then there are isomorphisms for $i \geqslant 1$,

$$
H_{N_{G}(A, c)}^{*}\left(c \times\left(F^{A}\right)^{i}\right) \stackrel{\cong}{\rightarrow} H_{C_{G}(A, c)}^{*}\left(c \times\left(F^{A}\right)^{i}\right)^{W_{G}(A, c)}
$$

Proof. Let $N=N_{G}(A, c), C+C_{G}(A, c), W=N / C$ and $Z=c \times\left(F^{A}\right)^{i}$ for a fixed $i \geqslant 1$.

Then $Z_{C}$ is a principal $W$-bundle over $Z_{n}$ and there is a Serre spectral sequence [B]

$$
H^{p}\left(B W,\left\{H_{C}^{q}(z)\right\}\right) \Rightarrow H_{N}^{p+q}(Z) .
$$

Now, $W$ is a finite group, so we may use results from the theory of cohomology of finite groups to compute $H^{p}\left(B W,\left\{H_{C}^{q}(Z)\right\}\right)$. By Lemma $3.5, H_{C}^{q}(Z)$ is a free $Z / p Z[W]$-module for $q \geqslant 0$, so one may conclude [C-E] that

$$
H^{p}\left(B W,\left\{H_{C}^{q}(Z)\right\}\right)=0 \text { if } p>0, q \geqslant 0 .
$$


Thus the above spectral sequence degenerates yielding

$$
H^{0}\left(B W,\left\{H_{C}^{*}\left(c \times\left(F^{A}\right)^{i}\right)\right\}\right) \stackrel{\cong}{\leftrightarrows} H_{N}^{*}\left(c \times\left(F^{A}\right)^{i}\right)
$$

or

$$
H_{C}^{*}\left(c \times\left(F^{A}\right)^{i}\right)^{W} \cong H_{N}^{*}\left(c \times\left(F^{A}\right)^{i}\right) \text {. Q.E.D. }
$$

Finally, we return to the

Proof of Theorem 3.2. Consider the following commutative diagram (it should be clear what the maps are):

$(*) \quad H_{G}^{*}(X)_{\mathfrak{p}_{(A, C)}} \rightarrow$

$$
\begin{aligned}
& H_{G}^{*}(X \times F)_{\mathfrak{p}_{(A, c)}} \quad \rightrightarrows \quad H_{G}^{*}\left(X \times F^{q}\right)_{\mathfrak{p}_{(4, c)}} \\
& H_{G}^{*}\left(G \cdot\left(c \times F^{A}\right)\right)_{\mathfrak{p}_{(A, C)}} \rightrightarrows H_{G}^{*}\left(G \cdot\left(c \times\left(F^{A}\right)^{2}\right)\right)_{\mathfrak{p}_{(A, C)}} \\
& \downarrow \text { (2) } \\
& H_{N_{(i}(A, c)}^{*}\left(c \times F^{A}\right)_{\mathfrak{p}_{(A, C)}} \rightrightarrows H_{N_{G(}(A, c)}^{*}\left(c \times\left(F^{A}\right)^{2}\right)_{\mathfrak{p}_{(A, C)}} \\
& \downarrow \text { (3) }
\end{aligned}
$$

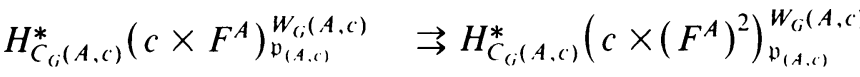

$$
\begin{aligned}
& \uparrow \text { (4) }
\end{aligned}
$$

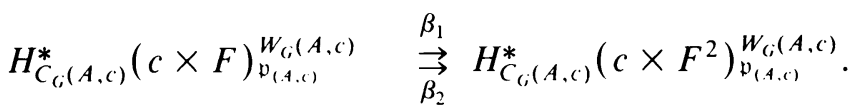

We observe:

(i) the sequence (*) is exact: this follows from the exact sequence of [Q1] plus exactness of localization;

(ii) the vertical arrows in each of squares 1 through 4 are isomorphisms:

Square 1. Apply Lemma 3.3 with $W=X$ and $K=G$.

Square 2. Apply Lemma 3.4, then localize.

Square 3. Apply Lemma 3.6, then localize.

Square 4. Apply Lemma 3.3 with $W=c$ and $K=C_{G}(A, c)$.

Since $(A, c)$ is maximal, it is "weakly closed" in $\mathscr{A}(K, W)$ in this case, and the isomorphism is isomorphism $(* *)$ of Lemma 3.3.

Thus we conclude that $\operatorname{ker}\left(\beta_{1}, \beta_{2}\right) \cong H_{G}^{*}(X)_{\mathfrak{p}_{(A, C)}}$. But, the exact sequence

$$
H_{C_{(i}(A, c)}^{*}(c) \rightarrow H_{C_{(i}(A, c)}^{*}(c \times F) \rightrightarrows H_{C_{C_{i}}(A, c)}^{*}\left(c \times F^{2}\right)
$$

of [Q1] plus exactness of localization of left exactness of taking invariants enables us to conclude that

$$
H_{C_{G}(A, c)}^{*}(c)_{p_{(, A, C)}}^{W_{G(A, c)}} \stackrel{\leftrightarrows}{\leftrightarrows} H_{G}^{*}(X)_{p_{(A, C)}} \text {. Q.E.D. }
$$

Let $H_{G}^{*}(X) \underset{r_{G, Y}}{\rightarrow} H_{N_{G}\left(A_{, c}\right)}^{*}(c)$ be the restriction map. If

$$
p_{(A, c)}=\operatorname{ker}\left(H_{G}(X) \rightarrow H_{A}(p t \in c) / \sqrt{0}\right)
$$


as usual, and

$$
\mathfrak{p}_{(A, c)}^{N}=\operatorname{ker}\left(H_{N_{G}(A, c)}(c) \rightarrow H_{A}(\mathfrak{p t} \in c) / \sqrt{0}\right)
$$

then $r_{G, N}^{-1}\left(\mathfrak{p}_{(A, C)}^{N}\right)=\mathfrak{p}_{(A, c)}$. Similarly, there is the prime $\mathfrak{p}_{(A, C)}^{C} \subseteq H_{C_{G}(A, C)}(c)$ and the restriction $H_{G}(X) \underset{r_{G, C}}{\rightarrow} H_{C_{G i}(A, c)}(c)$ with $r_{G, C}^{-1}\left(\mathfrak{p}_{(A, c)}^{C}\right) \doteq \mathfrak{p}_{(A, c)}$. Also we have $r_{N, C}^{-1}\left(\mathfrak{p}_{(A, C)}^{C}\right)=\mathfrak{p}_{(A, C)}^{N}$ where $H_{N_{G}(A, C)}(c) \underset{r_{N, C}}{\rightarrow} H_{C_{G}(A, C)}(c)$.

COROLlary 3.7. If $(A, c)$ is a maximal pair of $\mathscr{A}(G, X)$, then

$$
H_{G}^{*}(X)_{\mathfrak{p}_{(A, C)}} \stackrel{\cong}{\rightarrow} H_{N_{G}(A, c)}^{*}(c)_{\mathfrak{p}_{(A, C)}^{*}} \text {. }
$$

Proof. The main theorem gives an isomorphism

$$
H_{G}^{*}(X)_{\mathfrak{p}_{(A, C)}} \cong H_{C_{G}(A, C)}^{*}(c)_{\mathfrak{p}_{(A, C)}}^{W_{G(A, c)}} .
$$

Applied to $N_{G}(A, c)$ and $c$ instead of $G$ and $X$, it also gives an isomorphism

$$
H_{N_{G}(A, c)}^{*}(c)_{\mathcal{V}_{(A, C)}^{N}} \underset{\cong}{\cong} H_{C_{(i}(A, c)}^{*}(c)_{\mathfrak{p}_{(A, C)}^{N}}^{W_{G}(A, c)}
$$

Thus we need only show that

$$
H_{C_{G}(A, C)}^{*}(c)_{\mathfrak{V}_{(i, C)}^{*}}^{W_{G(A, C)}^{*}} \cong H_{C_{G}(A, C)}^{*}(c)_{(A, C)}^{W_{G(C)}(A, C)}
$$

or

$$
H_{C_{G(}(A, C)}^{*}(c)_{\mathfrak{p}_{(A, C)}^{N}} \cong H_{C_{G(}(A, C)}^{*}(c)_{\mathfrak{p}_{(A, C)}} .
$$

We do this by showing that both of the above rings are isomorphic to $H_{C_{G}(A, C)}^{*}(C)_{V(A)}$.

This follows from standard results in commutative algebra. Let $R_{G}=H_{G}(X)$, $R_{N}=H_{N_{G i}(A, c)}(c), R_{C}=H_{C_{G}(A, c)}(c)$ and $M=H_{C_{G(}(A, c)}^{*}(c)$. We may consider $M$ as an $R_{G^{-}}, R_{N^{-}}$or $R_{C}$-module, using the commutative double triangle:

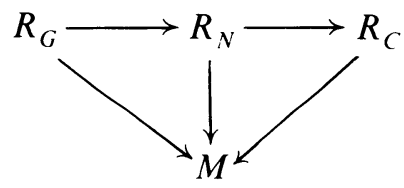

Now

$$
\begin{aligned}
M_{\mathfrak{p}} & =M \otimes_{R_{G}}\left(R_{G}\right)_{\mathfrak{p}}=M \otimes_{R_{G}}\left(R_{C}\right) \mathfrak{p} \quad \text { (by the main theorem) } \\
& =M \otimes_{R_{C}}\left(R_{C}\right)_{\mathfrak{p}}
\end{aligned}
$$

and

$$
\begin{aligned}
M_{\mathfrak{p}^{N}} & =M \otimes_{R_{N}}\left(R_{N}\right)_{\mathfrak{p}^{v}}=M \otimes_{R_{N}}\left(R_{C}\right)_{\mathfrak{W}^{v}} \quad \text { (by the main theorem) } \\
& =M \otimes_{R_{C}}\left(R_{C}\right)_{\mathfrak{p}^{v}} .
\end{aligned}
$$

(Here, $\mathfrak{p}=\mathfrak{p}_{(A, c)}, \mathfrak{p}^{C}=\mathfrak{p}_{(A, C)}^{C}$ and $\mathfrak{p}^{N}=\mathfrak{p}_{(A, C)}^{N}$ ) Thus, to prove the theorem it is enough to show that $\left(R_{C}\right)_{\mathfrak{p}}=\left(R_{C}\right)_{\mathfrak{p}}$ and $\left(R_{C}\right)_{\mathfrak{p}^{\mathrm{v}}}=\left(R_{C}\right)_{\mathrm{p}^{\mathrm{c}}}$.

There is a natural map $\left(R_{C}\right)_{\mathfrak{p}} \rightarrow\left(R_{C}\right)_{\mathfrak{p}}$ c since $r_{G, C}^{-1}\left(\mathfrak{p}^{C}\right)=\mathfrak{p}$. This map is an isomorphism if we show that $\left(R_{C}\right)_{\mathfrak{p}}$ is a local ring $[\mathbf{M}]$. If $\bar{a}$ is any prime in $\left(R_{C}\right)_{p}$ 
then $\bar{g}$ corresponds to a unique prime ideal a of $R_{C}$ disjoint from $r_{G, C}\left(R_{G}-\rho\right)$ (i.e., $\left.\mathfrak{g}_{\mathfrak{p}}=\overline{\mathfrak{q}}\right)$. Since $\mathfrak{g} \cap r_{G, C}\left(R_{G}-\mathfrak{p}\right)=\varnothing, r_{G, C}^{-1}(\mathfrak{g}) \cap R_{G}-\mathfrak{p}=\varnothing$, so $\mathfrak{p} \supseteq r_{G, C}^{-1}(\mathfrak{g})$. But $\mathfrak{p}$ is minimal in $R_{G}$ (using Quillen's characterization of minimal primes), so $\mathfrak{p}=$ $r_{G, C}^{-1}(g)$.

Now, $R_{G} / \mathfrak{p} \rightarrow R_{C} / \mathfrak{p}^{C}$ is integral $[\mathbf{Q} 1]$ and injective (since the triangle

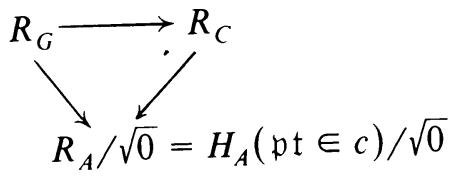

commutes), and both $\mathfrak{g} / \mathfrak{p}^{C}$ and the 0 -ideal in $R_{C} / \mathfrak{p}^{C}$ contract to the 0 -ideal in $R_{G} / \mathfrak{p}$; by the going-up theorem ([M], e.g.) we have $\mathrm{g} / \mathfrak{p}^{C}=0$ or $\mathfrak{p}^{C}=\mathrm{g}$. Thus, in fact, we have shown that $\overline{\mathrm{g}}=\left(\mathfrak{p}^{C}\right)_{\mathfrak{p}}$ is the only prime in $\left(R_{C}\right)_{\mathfrak{p}}$.

The same argument works for $R_{N}$ and $\mathfrak{p}^{N}$ in place of $R_{G}$ and $\mathfrak{p}$. Q.E.D.

In any noetherian ring the 0 -ideal may be decomposed as an intersection of primary ideals: $0=\mathfrak{g}_{1} \cap \cdots \cap \mathfrak{g}_{n}$. The set $\left\{\sqrt{\mathfrak{g}_{i}}\right\}$ is the set of associated primes of the ring. The minimal primes of the ring are the minimal elements of this set $\left\{\sqrt{\mathfrak{g}_{i}}\right\}$. The ideals of $\left\{g_{i}\right\}$ belonging to these minimal primes are called the isolated primary components; the rest are called embedded components. The isolated primary ideals are uniquely specified, the embedded components are not. The following theorem characterizes the isolated primary components of $H_{G}(X)$.

THEOREM 3.8. If $G$ is finite, the isolated primary ideals of $H_{G}(X)$ are of the form

$$
\mathrm{g}_{(A, c)}=\operatorname{ker}\left(H_{G}(X) \rightarrow H_{C_{G}(A, c)}(c)\right),
$$

where $(A, c)$ is a maximal pair of $\mathscr{A}(G, X)$.

Proof. We need only show that if $(A, c)$ is maximal then $\mathfrak{g}_{(A, c)}$ is $\mathfrak{p}_{(A, c)}$-primary; i.e., $\sqrt{\mathfrak{g}_{(A, c)}}=\mathfrak{p}_{(A, c)}$ and $\mathfrak{g}_{(A, c)}$ is primary (in view of Quillen's characterization of the minimal primes).

Consider the map

$$
H_{G}(X) \stackrel{r_{G, C}}{\rightarrow} H_{C_{G}(A, c)}(c)
$$

Let $\mathfrak{p}^{C}=\mathfrak{p}_{(A, c)}^{C}$ and $\mathfrak{p}=\mathfrak{p}_{(A, c)}$ be as above. Since $\mathfrak{p}^{C}$ is the only associated prime of $H_{C_{G}(A, c)}(c)$ (this ring is Cohen-Macaulay by [D1] so has no embedded primes, and $\mathfrak{p}^{C}$ is the only minimal prime by [Q1]) we see that 0 is $\mathfrak{p}^{C}$-primary (uniqueness of primary decomposition). Thus $r_{(G, C)}^{-1}(0)$ is $r_{G, C}^{-1}\left(\mathfrak{p}^{C}\right)$-primary-i.e., $\mathfrak{g}_{(A, c)}$ is $\mathfrak{p}$-primary. Q.E.D.

REMARK. This theorem should be true for $G$ compact Lie, but the results of [D1] needed are proved only for finite groups at this point.

It is perhaps of interest to see what these theorems say when $X$ is a one-point space. Then $H_{G}^{*}(X)$ is group cohomology- $H^{*}(B G)$. For example, Theorem 3.2 gives an isomorphism

$$
H^{*}(B G)_{\mathfrak{p}_{A}} \rightarrow H^{*}\left(B\left(C_{G}(A)\right)\right)_{\mathfrak{p}_{A}}^{W_{G}(A)}
$$


where $\mathfrak{p}_{A}=\operatorname{ker}\left(H^{*}(B G) \rightarrow H^{*}(B A) / \sqrt{0}\right)$ is the prime belonging to a maximal $p$-torus $A$ of $G$. In the next section we give an application of this result.

4. An example. Let $\mathrm{GL}_{n}(\mathbf{Z} / p \mathbf{Z})=\mathrm{GL}_{n}$ denote the group of invertible $n \times n$ matrices over $\mathbf{Z} / p \mathbf{Z}$; or equivalently the group of invertible linear maps of an $n$-dimensional vector space over $\mathbf{Z} / p \mathbf{Z}$. Consider the subgroup of $\mathrm{GL}_{n}$ given by

$$
A_{1}=\left\{\left[\begin{array}{cccc}
1 & a_{1} & \cdots & a_{n-1} \\
& 1 & & \bigcirc \\
& \bigcirc & \ddots & \\
& & & 1
\end{array}\right] \mid a_{i} \in \mathbf{Z} / p \mathbf{Z}, i=1,2, \ldots, n-1\right\} .
$$

This subgroup is a $p$-torus in $\mathrm{GL}_{n}$. One can compute its centralizer

$$
\begin{aligned}
C_{\mathrm{GL}_{n}}\left(A_{1}\right) & =\left\{\left[\begin{array}{cccc}
d & a_{1} & \cdots & a_{n-1} \\
& d & & \bigcirc \\
& \bigcirc & \ddots & \\
& & d
\end{array}\right] \mid d \in(\mathbf{Z} / p \mathbf{Z})^{*}\right\} \\
& \cong(\mathbf{Z} / p \mathbf{Z})^{*} \times A_{1}
\end{aligned}
$$

(here, $(\mathbf{Z} / p \mathbf{Z})^{*}$ is the group of unit in $\mathbf{Z} / p \mathbf{Z}$ of order $\left.p-1\right)$, and its normalizer, the parabolic subgroup

$$
\begin{aligned}
N_{\mathrm{GL}_{n}}\left(A_{1}\right)=P_{1}=\left\{\left[\begin{array}{c|ccc}
x & a_{1} & \cdots & a_{n-1} \\
\hdashline & & Y
\end{array}\right] \mid x \in(\mathbf{Z} / p \mathbf{Z})^{*}=\mathrm{GL}_{1}, Y \in \mathrm{GL}_{n-1}\right. \\
\\
\left.\quad \text { and } a_{i} \in \mathbf{Z} / p \mathbf{Z}, i=1,2, \ldots, n-1\right\} .
\end{aligned}
$$

Since the index of $A_{1}$ in its centralizer has order prime to $p$, we see that $A_{1}$ is maximal p-torus of $\mathrm{GL}_{n}$. The "Weyl" group of $A_{1}, W_{\mathrm{GL}_{n}}\left(A_{1}\right)=P_{1} /\left(A_{1} \times(\mathbf{Z} / p \mathbf{Z})^{*}\right)$ is contained in the automorphism group of $A_{1}$, which is naturally isomorphic to $\mathrm{GL}_{n-1}$. One sees that this inclusion $W_{\mathrm{GL}_{n}}\left(A_{1}\right) \hookrightarrow \operatorname{Aut}\left(A_{1}\right)$ is, in fact, an isomorphism (by computing orders, for example); also, the action of $W_{\mathrm{GL}_{n}}\left(A_{1}\right)$ on $A_{1}$ is equivalent to the natural action of $\mathrm{GL}_{n-1}$ on $A_{1}$ (where $A_{1}$ is regarded as being an $(n-1)$ dimensional vector space over $\mathbf{Z} / p \mathbf{Z}$ ).

Using the Künneth formula, one computes

$$
H^{*}\left(C_{\mathrm{GL}_{n}}\left(A_{1}\right)\right)=H^{*}\left(A_{1}\right) \otimes_{\mathbf{Z} / p \mathbf{Z}} H^{*}\left((\mathbf{Z} / p \mathbf{Z})^{*}\right),
$$

since $\left|(\mathbf{Z} / p \mathbf{Z})^{*}\right|=p-1$ is prime to $p$, its mod-p cohomology is zero in positive degrees, so that

$$
H^{*}\left(C_{\mathrm{GL}_{n}}\left(A_{1}\right)\right)=H^{*}\left(A_{1}\right) \text {. }
$$

This last ring is well known:

$$
H^{*}\left(A_{1}, \mathbf{Z} / p \mathbf{Z}\right) \cong\left\{\begin{array}{l}
\mathbf{Z} / p \mathbf{Z}\left[a_{1}, \ldots, a_{n-1}\right] \otimes_{\mathbf{Z} / p \mathbf{Z}} \wedge\left[b_{1}, \ldots, b_{n-1}\right], \quad p>2, \\
\mathbf{Z} / p \mathbf{Z}\left[a_{1}, \ldots, a_{n-1}\right], \quad p=2
\end{array}\right.
$$


(i.e., either a polynomial algebra tensored with an exterior algebra, or a polynomial algebra).

We assume that $p$ is odd from now on.

Mui [Mui] has computed the ring of invariants

$$
R^{\mathrm{GL}_{n-1}}=\left(\mathbf{Z} / p \mathbf{Z}\left[a_{1}, \ldots, a_{n-1}\right] \otimes_{\mathbf{Z} / p \mathbf{Z}} \bigwedge\left[b_{1}, \ldots, b_{n-1}\right]\right)^{\mathrm{GL}_{n-1}}
$$

it is generated by $Q_{0}, \ldots, Q_{n-2}\left(n-1\right.$ polynomial generators) and $R_{s_{1}, s_{2} \ldots, s_{k}}$, where $1 \leqslant k \leqslant n-1$ and $0 \leqslant s_{1}<s_{2}<\cdots<s_{k} \leqslant n-2$ is a selection of $k$ numbers from the set $\{0,1,2, \ldots, n-2\}$ (thus there are $2^{n-1}-1$ of these generators).

The relations are $R_{s}^{2}=0$ for every $s$ between 0 and $n-2$ and $R_{s_{1}} R_{s_{2}} \cdots R_{s_{k}}=$ $(-1)^{k(k-1) / 2} R_{s_{1}, \ldots s_{k}} Q_{0}^{k-1}$ for every $k$ between 1 and $n-1$, and every subset of $k$ elements $s_{1}<s_{2}<\cdots<s_{k}$ of $\{0,1, \ldots, n-2\}$.

Since $Q_{0}$ is not a zero divisor in this invariant subring (it is not a zero divisor in $\left.R \supseteq R^{\mathrm{GL}_{n-1}}\right)$, one sees that the above two relations imply that $R_{s_{1} \ldots s_{h}}^{2}=0$ for every set of indices $\left\{s_{1}, \ldots, s_{k}\right\}$.

The degrees of the generators are as follows:

$$
\text { degree }\left(Q_{i}\right)=2\left(p^{n-1}-p^{i}\right), \quad i=0,1, \ldots, n-2,
$$

and

$$
\operatorname{degree}\left(R_{s_{1}, \ldots s_{k}}\right)=k+2\left(p^{n-1}-1\right)-2\left(p^{s_{1}}+\cdots+p^{s_{k}}\right) .
$$

There is a unique minimal prime $\mathfrak{p}$ in the even part of this ring, namely, the nilpotent elements, or the ideal generated by the $R$ 's. Localizing at this prime means inverting all the $Q_{i}$ 's. Letting $P_{i}=R_{i} / Q_{0}$ for $0 \leqslant i \leqslant n-2$ ("degree" $\left(P_{i}\right)=1-$ $2 p^{i}$ ) one sees that the relations in $R$ imply that $P_{i}^{2}=0$ and that

$$
R_{\mathfrak{p}}^{\mathrm{GL}}{ }_{n-1}=\mathbf{Z} / p \mathbf{Z}\left(Q_{0}, \ldots, Q_{n-2}\right) \otimes_{\mathbf{z} / p \mathbf{Z}} \wedge\left(P_{0}, \ldots, P_{n-2}\right),
$$

i.e., the quotient field of the polynomial $\operatorname{ring} \mathbf{Z} / p \mathbf{Z}\left[Q_{0}, \ldots, Q_{n-2}\right]$ tensored with an exterior algebra on the $P_{i}$ 's.

Now, since

$$
H^{*}\left(C_{\mathrm{GL}_{n}}\left(A_{1}\right)\right)_{\mathfrak{p}_{A_{1}}}^{W_{\left(i l_{n}\right.}\left(A_{1}\right)}=k\left(Q_{0}, \ldots, Q_{n-2}\right) \otimes \wedge\left(P_{0}, \ldots, P_{n-2}\right)
$$

(use the preceding paragraphs and the argument used in the proof of Corollary 3.7), the main theorem of $\$ 3$ implies that

$$
H^{*}\left(\mathrm{GL}_{n}(\mathbf{Z} / p \mathbf{Z}), \mathbf{Z} / p \mathbf{Z}\right)_{\mathfrak{p}_{A_{1}}} \cong k\left(Q_{0}, \ldots, Q_{n-2}\right) \otimes \wedge\left(P_{0}, \ldots, P_{n-2}\right)
$$

(here $k=\mathbf{Z} / p \mathbf{Z}$ ).

\section{REFERENCES}

[B] A. Borel et al., Seminar on compact transformation groups, Ann. of Math. Studies, vol. 46, Princeton Univ. Press, Princeton, N. J., 1960.

[Br] G. Bredon, Introduction to compact transformation groups, Academic Press, New York, 1972.

[C-E] H. Cartan and S. Eilenberg, Homological algebra, Princeton Univ. Press, Princeton, N. J., 1954.

[D1] J. Duflot, Depth and equivariant cohomologv, Comment. Math. Helv. 56 (1981), 627-637.

[H] Wu-Yi Hsiang, Cohomolog theory of topological transformation groups, Springer-Verlag, New York, 1975. 
[M] H. Matsumura, Commutative algebra, Benjamin, New York, 1970.

[Mui] H. Mui, Modular intariant theory and cohomology algebras of symmetric groups, J. Fac. Sci. Univ. Tokyo Sect. IA Math 22 (1975), 319-369.

[Q1, Q2] D. Quillen. The spectrum of an equivariant cohomology ring. I, II, Ann. of Math. (2) 94 (1971), 549-602.

[T] T. tom Dieck, Transformation groups and representation theory, Lecture Notes in Math., vol. 766, Springer-Verlag, Berlin and New York, 1979.

Department of Mathematics, Brandeis University, Waltham, Massachusetts 02354 (Current address)

Department of Mathematics, Colorado State University, Fort Collins, Colorado 80523 